\title{
Cost-Related Medication Non-adherence, Cost Coping Behaviors, and Cost Conversations Among Individuals with and Without Diabetes
}

J Gen Intern Med 36(9):2867-9

DOI: $10.1007 / \mathrm{s} 11606-020-06176-4$

(c) Society of General Internal Medicine 2020

\section{INTRODUCTION}

Patients with chronic conditions, particularly diabetes mellitus, are at high risk for cost-related medication nonadherence. ${ }^{1,2}$ Cost coping behaviors, including cutting back on basic needs, skipping doses of medication, or asking for samples, have been documented among patients with chronic diseases. ${ }^{1,3}$ Some of these behaviors, particularly cutting back on basic needs such as food, could be detrimental to diabetes management. ${ }^{4}$ Physicians may be aware that cost coping occurs; however, they may not have cost conversations with patients even when indicated. ${ }^{2,5,6} \mathrm{We}$ compared cost coping behaviors and cost conversations among individuals with and without diabetes who have multiple chronic conditions.

\section{METHODS}

We consented 270 participants (81 with diabetes and 189 without diabetes) recruited through community primary care clinics, an academic multi-specialty clinic, and local newspaper ad in St. Louis, MO, for one survey, administered by a trained research assistant, between 12/21/2016 and 12/06/ 2017. Individuals were eligible if they were aged $35-80$ years, English-speaking, with at least one chronic health condition, and taking at least one medication. The study was approved by the Washington University Human Research Protection office on 03/09/2016. Survey topics included demographics, healthcare utilization and literacy, medications and diagnoses, financial strain, and provider communication. We conducted bivariate comparisons of participants with and without selfreported diabetes using Student's $t$ test for continuous variables and chi-square or Fisher's exact test for categorical variables. We examined diabetes as an independent risk factor

$\overline{\text { Prior Presentations Preliminary findings from this study were reported }}$ at the 79th Scientific Sessions of the American Diabetes Association from June 7 to 11, 2019, in San Francisco, CA.

Received May 15, 2020

Accepted August 20, 2020

Published online September 1, 2020 for spending less on basic needs to pay for medication in a multivariable logistic regression model.

\section{RESULTS}

There were no statistically significant differences between participants with and without diabetes on sex, race/ethnicity, or marital status, but those with diabetes were older than individuals without diabetes $(58.4$ v. 53.8 years, $p<0.001)$ (Table 1). Both groups were majority Black non-Hispanic (> $80 \%$ ) and had multiple comorbidities. Compared with individuals without diabetes, a lower proportion of individuals with diabetes had monthly income $<\$ 800(33.8 \%$ v. $51.3 \%$, $p=0.008)$ and a higher proportion had private insurance or Medicare $(40.7 \%$ v. $22.8 \%, p=0.003)$. Individuals with diabetes had higher estimated medication costs and were more likely to use medical supplies and to have asked for and received medication samples. On financial strain measures, individuals with diabetes were more likely to report spending less on basic needs to pay for medications $(58.0 \%$ v. $41.8 \%$, $p=0.01)$ and have filed for bankruptcy (38.3\% v. $21.7 \%, p=$ 0.005). Among those spending less on basic needs to afford medications, almost $80 \%$ reported spending less on food and groceries. In the multivariable logistic regression model adjusted for age, sex, race/ethnicity, \# of other comorbidities, and insurance status, individuals with diabetes remained more likely to report spending less on basic needs to pay for medication than those without diabetes (aOR 1.78 (95\% CI 1.013.14)) (Table 2).

A higher proportion of individuals with diabetes reported talking with a doctor about affordability of healthcare or medications $(66.7 \%$ v. $49.2 \%, p=0.008)$, and a large majority of all participants reported they were comfortable talking to their doctor about medication cost. Notably, individuals with diabetes were less comfortable than those without diabetes discussing cost with a receptionist, medical assistant, or pharmacy technician (Table 1).

\section{DISCUSSION}

To our knowledge, this is the first report of cost coping and cost conversation strategies among individuals with multiple chronic conditions, comparing participants with and without 
Table 1 Bivariate comparison of survey participants with and without self-reported diabetes $(n=270)$ in St. Louis, MO, 2016-2017

\begin{tabular}{|c|c|c|c|}
\hline & $\begin{array}{l}\text { Diabetes mean (SD) or } n \\
(\%) \\
n=81\end{array}$ & $\begin{array}{l}\text { No diabetes mean (SD) or } n \\
(\%) \\
n=189\end{array}$ & $p^{*}$ \\
\hline \multicolumn{4}{|l|}{ Demographics/comorbidities } \\
\hline Age (years) & $58.4(9.5)$ & $53.8(9.4)$ & $\begin{array}{l}<.001^{\S} \\
0\end{array}$ \\
\hline Sex (female) & $54(66.7)$ & $107(56.6)$ & 0.12 \\
\hline Race/ethnicity (non-Hispanic Black) & $65(80.3)$ & $156(82.5)$ & 0.65 \\
\hline Marital status (married/partner) & $16(19.8)$ & $35(18.5)$ & 0.54 \\
\hline Monthly income $(<\$ 800)$ & $27(33.8)$ & $96(51.3)$ & $0.008^{\ddagger}$ \\
\hline Insurance status (private/Medicare) & $33(40.7)$ & $43(22.8)$ & $0.003^{\ddagger}$ \\
\hline \# of comorbidities (excluding diabetes) & $4.8(2.6)$ & $4.3(2.3)$ & 0.15 \\
\hline \multicolumn{4}{|l|}{ Medication/supply use } \\
\hline Use of medical supplies & $63(77.8)$ & $38(20.1)$ & $\begin{array}{l}< \\
0.001^{\S}\end{array}$ \\
\hline Skipped doses (always/often/sometimes) & $23(28.4)$ & $41(21.7)$ & 0.24 \\
\hline Asked for/received samples & $42(51.9)$ & $55(29.1)$ & $<.001^{\S}$ \\
\hline \multicolumn{4}{|l|}{ Financial strain } \\
\hline Spent less on basic needs to pay for medications & $47(58.0)$ & $79(41.8)$ & $0.01^{\dagger}$ \\
\hline Spent less on food/groceries $(n=126)$ & $37(78.7)$ & $64(81.0)$ & 0.76 \\
\hline Filed for bankruptcy & $31(38.3)$ & $41(21.7)$ & $0.005^{+}$ \\
\hline Medication cost change month-to-month & $20(25.3)$ & $26(14.9)$ & 0.05 \\
\hline Estimated total spent on medications in the last month & $\$ 91.99(\$ 160.55)$ & $\$ 42.18(\$ 82.47)$ & $0.001^{\S}$ \\
\hline \multicolumn{4}{|l|}{ Communication with healthcare providers about cost } \\
\hline $\begin{array}{l}\text { Ever talked with a doctor about affordability of healthcare/ } \\
\text { medications }\end{array}$ & $54(66.7)$ & $93(49.2)$ & $0.008^{\ddagger}$ \\
\hline \multicolumn{4}{|l|}{ Comfortable talking with healthcare professional about medications } \\
\hline Doctor & $70(86.4)$ & $157(83.1)$ & 0.49 \\
\hline $\mathrm{PA} / \mathrm{NP}$ & $50(61.7)$ & $132(69.8)$ & 0.19 \\
\hline Nurse & $49(60.5)$ & 135 (71.4) & 0.08 \\
\hline Pharmacist & $52(64.2)$ & $136(72.0)$ & 0.20 \\
\hline Social worker/case manager & $60(74.1)$ & $140(74.1)$ & 1.00 \\
\hline Receptionist & $17(21.0)$ & $63(33.3)$ & $0.04^{\dagger}$ \\
\hline Medical assistant & $32(39.5)$ & $109(57.7)$ & $0.006^{\ddagger}$ \\
\hline Pharmacy technician & $33(40.7)$ & $109(57.7)$ & $0.01^{\dagger}$ \\
\hline Office manager & $26(32.1)$ & $68(36.0)$ & 0.54 \\
\hline Billing/other financial person & $32(39.5)$ & $92(48.7)$ & 0.17 \\
\hline
\end{tabular}

*Student's t test for continuous variables and chi-square or Fisher's exact test for categorical variables for bivariate comparisons of participants with and without self-reported diabetes

$t p<0.05 ;{ }^{t} p<0.01 ;{ }^{s} p<0.001$

diabetes. Most strikingly, while individuals with diabetes were more likely to have private insurance or Medicare, they were still more likely to report spending less on basic needs to pay for medications. Individuals commonly spent less on food, a trade off that can be counterproductive for type 2 diabetes where healthy food choices may improve glycemic control and minimize medication need. ${ }^{4}$ However, healthy foods may also be more expensive and difficult to access than generic medications. Our study highlights the importance of considering multi-modal interventions for individuals with diabetes and financial strain, addressing not only medication cost but also food insecurity and quality. Cost conversations are important and acceptable within the clinical encounter and must address basic needs as well as medication. Future studies should elucidate the tradeoffs between food, medical supplies, and medications among individuals with diabetes and assess the acceptability and impact of novel methods to address cost coping.

Table 2 Unadjusted and adjusted analyses assessing factors associated with spending less on basic needs to pay for medications $(n=270)$ in St. Louis, MO, 2016-2017

\begin{tabular}{lll}
\hline \hline & Unadjusted OR (95\% CI) & Adjusted OR (95\% CI)* \\
\hline Diabetes (Ref=no diabetes) & $1.93(1.14-3.26)$ & $1.78(1.01-3.14)^{\dagger}$ \\
\# other comorbidities & $1.26(1.13-1.41)$ & $1.25(1.11-1.40)^{\S}$ \\
Age (years) & $1.01(0.98-1.03)$ & $0.99(0.96-1.02)$ \\
Female sex (Ref=male) & $1.63(1.00-2.67)$ & $1.34(0.78-2.28)$ \\
Race/ethnicity (Ref=non-Hispanic White) & $0.73(0.39-1.36)$ & $0.98(0.50-1.92)$ \\
Insurance status (Ref=private/Medicare) & $0.67(0.39-1.13)$ & $0.78(0.43-1.41)$ \\
\hline
\end{tabular}

* Results from multivariable logistic regression

t $p<0.05 ;{ }^{*} p<0.01 ;{ }^{s} p<0.001$

IIThe dichotomous insurance status variable compared individuals on Medicaid, Gateway to Better Health (safety net program in St. Louis city and county), other public coverage or no coverage to individuals with private insurance or Medicare 
Acknowledgments: We would like to acknowledge all study participants and our community partner, Affinia Healthcare. We would also like to thank Miquela Ibrao, MSW, MPH, Lindsey Manshack, MPH, Natasan McCray, MHA, Amanda Lee, MPH, and Jessica Hao, MD for contributing to data collection.

Cynthia J. Herrick, MD, MPHS ${ }^{1,2}$

Sarah Humble, $\mathrm{MS}^{2}$

Laura Hollar, $M D^{3}$

Su-Hsin Chang, $\mathrm{PhD}^{2}$

Jean Hunleth, $\mathrm{PhD}, \mathrm{MPH}^{2}$

Amy McQueen, $\mathrm{PhD}^{4}$

Aimee S. James, $\mathrm{PhD}, \mathrm{MPH}^{2}$

${ }^{1}$ Department of Medicine, Division of Endocrinology, Metabolism and Lipid Research, Washington University School of Medicine,

St. Louis, MO, USA

${ }^{2}$ Department of Surgery, Division Public Health Sciences, Washington University School of Medicine,

St. Louis, MO, USA

${ }^{3}$ Heritage Medical Associates,

Nashville, TN, USA

${ }^{4}$ Department of Medicine, Division of General Medical Sciences, Washington University School of Medicine,

St. Louis, MO, USA

Corresponding Author: Cynthia J. Herrick, MD, MPHS; Department of Medicine, Division of Endocrinology, Metabolism and Lipid Research, Washington University School of Medicine, St. Louis, MO, USA (e-mail: herrickc@wustl.edu).

Funding Information This research was support by grant R01MD010445 (PI: Aimee James), from the National Institute on Minority Health and Health Disparities. Use of REDCap was supported by a Clinical and Translational Science Award (CTSA)
Grant (UL1TRO00448) and Siteman Comprehensive Cancer Center and NCI Cancer Center Support Grant P30CA091842. Dr. McQueen is also supported by R01DK115916 from the National Institute of Diabetes and Digestive and Kidney Diseases. Dr. Herrick is currently supported on a career development award K23HD096204 from the National Institute of Child Health and Human Development and was previously funded through KL2TRO02346 from the National Center for Advancing Translational Sciences. Dr. Hollar received funding from T32DK007120 through the National Institute of Diabetes and Digestive and Kidney Diseases.

\section{Compliance with Ethical Standards:}

The study was approved by the Washington University Human Research Protection office on 03/09/2016.

Conflict of Interest: None of the members of the research team have other financial conflicts of interest to disclose.

\section{REFERENCES}

1. Cohen RA, Cha AE. Strategies Used by Adults With Diagnosed Diabetes to Reduce Their Prescription Drug Costs, 2017-2018. NCHS Data Brief. 2019; 349:1-8.

2. Herkert D, Vijayakumar P, Luo J, et al. Cost-Related Insulin Underuse Among Patients With Diabetes. JAMA Intern Med. 2019;179(1):112-4.

3. Reed M, Brand R, Newhouse JP, Selby JV, Hsu J. Coping with prescription drug cost sharing: knowledge, adherence, and financial burden. Health Serv Res. 2008; 43(2):785-97.

4. Seligman HK, Jacobs EA, Lopez A, Tschann J, Fernandez A. Food insecurity and glycemic control among low-income patients with type 2 diabetes. Diabetes Care. 2012; 35(2):233-8.

5. Perez SL, Weissman A, Read S, et al. U.S. Internists' Perspectives on Discussing Cost of Care With Patients: Structured Interviews and a Survey. Ann Intern Med. 2019; 170(9_Suppl):S39-S45.

6. Bradham DD, Garcia D, Galvan A, Erb C. Cost-of-Care Conversations During Clinical Visits in Federally Qualified Health Centers: An Observational Study. Ann Intern Med. 2019; 170(9_Suppl):S87-S92.

Publisher's Note: Springer Nature remains neutral with regard to jurisdictional claims in published maps and institutional affiliations. 